\title{
Vladislav V. Levshinskii \\ Multiclass Classification in the Problem of Differential Diagnosis of Venous Diseases Based on Microwave Radiometry Data
}

Abstract. This article is devoted to applying mathematical models in the differential diagnosis of venous diseases based on microwave radiometry data. A modified approach for transforming feature space in thermometric data is described. After constructing features, a multiclass classification problem is solved in several ways: by reducing to binary classification problems using "one versus rest" and "one versus one" methods and building a multivariate logistic regression model. The best classification model achieved an average balanced accuracy score of 0.574 . A key feature of the approach is that classification result can be explained and justified in terms understandable to a diagnostician. This article presents the most significant patterns in thermometric data and the accuracy with which they can identify different classes of diseases.

Key words and phrases: microwave radiometry, mathematical modeling, feature construction, multiclass classification.

2010 Mathematics Subject Classification: 97M60; 68T30, 68T35

\section{Introduction}

Nowadays, it is incredibly relevant to develop intelligent systems based on applying various methods of artificial intelligence [1]. Such systems can help to interpret and analyze examination data and support decision making in medical diagnosis. Systems of the greatest interest are advisory systems that apply artificial intelligence methods and algorithms and contain mechanisms to explain the proposed solutions. The development of advisory systems requires using mathematical modeling, machine learning, and data analysis methods.

The reported study was funded by RFBR, project number 19-31-90153.

(c) V. V. LeVshinskit, 2021

(C) Volgograd State University, 2021

(C) Program Systems: Theory and Applications (design), 2021

10.25209/2079-3316-2021-12-2-37-52 
Microwave radiometry is a promising diagnostic method based on the examination of intrinsic electromagnetic radiation of human tissues in the microwave and infrared wavelength ranges. Its vital feature is absolute harmlessness to the patient. The method is successfully applied in various fields of medicine [2-4], in particular, in the early diagnosis and dynamic control of varicose diseases of the lower extremities [5] classified as "diseases of civilization," since the number of people suffering from them is estimated in billions.

Examination technique consists of consecutive measurement of internal and surface (skin) temperatures, registration of temperatures in numerical data, and subsequent examination data analysis. A specialist search for anomalies in thermometric data is a highly complex intellectual task requiring long training and many years of experience. Future development of intelligent systems will both improve the quality of diagnosis in general and also solve the problem of lack of narrow specialists, which will make possible the mass application of this method. Interpretation and formalization of expert knowledge and knowledge extraction from the data are critical stages in developing models for solving such problems.

During the last decade, the first studies have appeared on applying mathematical modeling, machine learning, and data analysis to diagnose varicose diseases of the lower extremities based on microwave radiometry data. The first models were based on Bayesian classifier [6]. Feature space consisted of temperature values, and the criteria used in making the diagnosis were incomprehensible to a diagnostician. All this created significant difficulties in justifying and explaining a diagnostic decision.

Statistical models have become prerequisites for the creation of effective models and algorithms that allow interpretation and justification of result $[7,8]$. Those models were applied to solve the binary classification problem Healthy/Sick.

In a related field, in the diagnosis of breast cancer based on microwave radiometry data, as a result of data mining, a significant number of patterns describing anomalies in the behavior of temperature fields have been revealed [9]. They are the basis for a model that allows to justify and explain a result not only in the diagnosis of breast cancer but also in the diagnosis of venous diseases $[\mathbf{1 0}, \mathbf{1 1}]$.

The purpose of this study is to apply the model for dynamically describing a patient condition in the problem of differential diagnosis of venous diseases based on microwave radiometry data.

Of course, the problem of differential diagnosis is not considered for the first time. Previously, D.A. Vedenyapin and A.G. Losev applied neural 


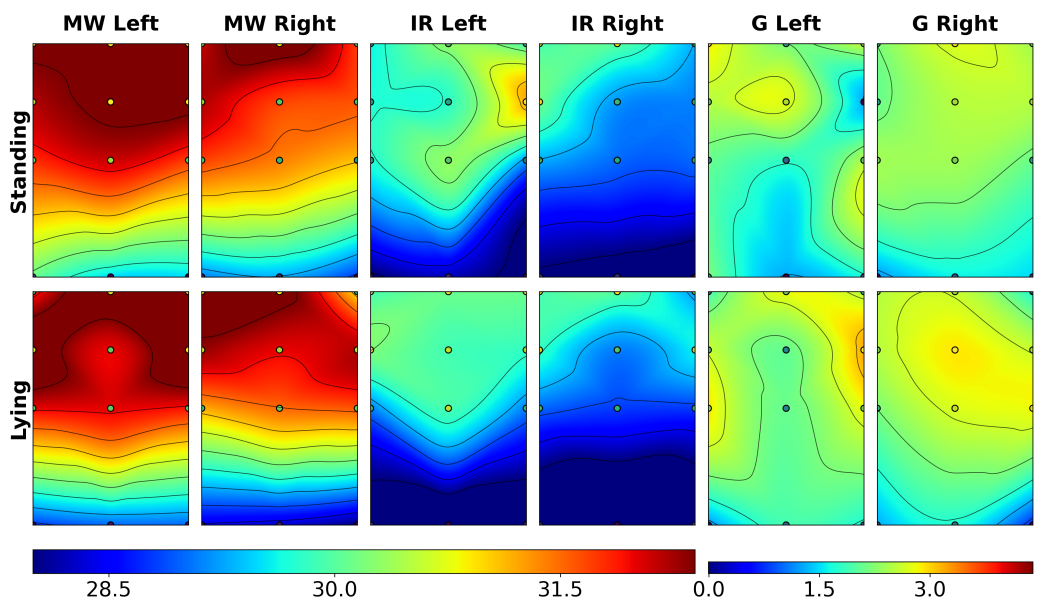

Figure 1. Temperature fields of a patient whose left leg is affected by venous disease. MW is internal, IR is surface temperatures and $\mathrm{G}$ is internal temperature gradients. The data is interpolated using cubic splines.

networks [12] to solve it. There is a comparative review of that approach in the conclusion.

\section{Data and methods}

\subsection{Microwave radiometry}

Microwave radiometry is a biophysical non-invasive examination method consisting of the consecutive measurement of internal and surface temperatures at specific points and registration of temperatures in numerical data. A specialist analyzes examination data in thermograms or maps of temperature fields to detect temperature anomalies and conclude the state of health or the need for further examinations. A method is based on the fact that temperature anomalies precede structural changes.

As an example, Figure 1 shows maps of the internal and surface temperature fields of a patient whose left leg is affected by venous disease.

During the examination of the lower extremities, a specialist measures the internal and surface temperatures at 12 symmetrical points located along the back surface of both lower legs, according to Figure 2. Several measurements are being taken for a patient in different positions: lying on the stomach and standing up. 


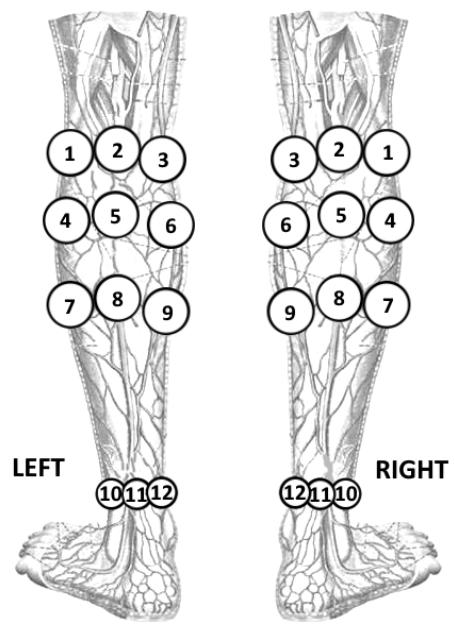

Figure 2. Sampling points on each leg (1-12).

\subsection{Dataset}

Dataset containing measurement data of the lower extremities of 146 patients (292 lower legs) is being analyzed. Each lower leg is labeled depending on the presence of a particular disease:

0 (Healthy) is measurement data of the legs without diseases, 36 lower legs $(12.3 \%)$

1 (Norm 2) is healthy lower legs of patients with venous disease on the other lower leg, 67 lower legs $(22.9 \%)$;

$2(C V I)$ is lower legs with chronic venous insufficiency, $100(34.2 \%)$;

3 (PTS) is post-thrombotic syndrome, 69 lower legs (23.6\%);

$4(A D V T)$ is acute deep vein thrombosis, $20(6.8 \%)$.

Formally, the dataset can be represented as a matrix

$$
X=\left[\begin{array}{cccc}
t_{1}^{1} & t_{2}^{1} & \ldots & t_{n}^{1} \\
t_{1}^{2} & t_{2}^{2} & \ldots & t_{n}^{2} \\
\ldots \ldots & \ldots . . & \ldots \\
t_{1}^{m} & t_{2}^{m} & \ldots & t_{n}^{m}
\end{array}\right], y=\left[\begin{array}{c}
y_{1} \\
y_{2} \\
\ldots \\
y_{m}
\end{array}\right], Y=\{1,2, \ldots, C\},
$$

where $m$ is the number of objects in the dataset, $n$ is the number of features, $x^{i}=\left(t_{1}^{i}, \ldots, t_{n}^{i}\right)$ is the feature vector of object $i, Y$ is the set of class labels and $y_{i} \in Y$ is a class label. 


\subsection{Feature construction}

The process of feature constructing and building a model for dynamically describing condition of each lower leg consists of several steps.

Feature vector contains 48 values of internal and surface temperatures measured at certain points of the lower legs in the lying and standing positions. Measurement points are shown in Figure 2. At the first step, temperature data is being split into the following groups:

1. Internal temperatures, standing position

$$
T^{i, m w, s t}=\left(T_{1}^{i, m w, s t}, \ldots, T_{12}^{i, m w, s t}\right)
$$

2. Internal temperatures, lying position

$$
T^{i, m w, l y}=\left(T_{1}^{i, m w, l y}, \ldots, T_{12}^{i, m w, l y}\right)
$$

3. Surface temperatures, standing position

$$
T^{i, i r, s t}=\left(T_{1}^{i, i r, s t}, \ldots, T_{12}^{i, i r, s t}\right)
$$

4. Surface temperatures, lying position

$$
T^{i, i r, l y}=\left(T_{1}^{i, i r, l y}, \ldots, T_{12}^{i, i r, l y}\right)
$$

Here, superscript $m w$ or $i r$ indicates the range of temperatures (internal or surface), and superscript st or ly (standing or lying) indicates the patient position during the measurement. Subscript is a point number.

There is an additional special group called internal gradients. That group contains differences between internal and surface temperatures at the corresponding points. For example, gradients of internal temperatures measured in the lying position are represented as

$$
T^{i, g, l y}=\left(T_{1}^{i, g, l y}, \ldots, T_{12}^{i, g, l y}\right) .
$$

Further, for every group of points and separately for pairs of groups, several valuable characteristics are calculated. These characteristics are presented in the form of hypotheses about the behavior of temperature fields and the corresponding generalized mathematical descriptions $[9,10]$ :

1. Hypothesis about an insignificant temperature difference, according to which healthy lower legs are characterized by low values of the following functionals:

1.1. Temperature oscillation

$$
F_{1}(T)=\max _{t \in T} t-\min _{t \in T} t
$$

where $T$ is a set of temperatures. 
1.2. Temperature deviation

$$
F_{2}(T)=S T_{\text {dev }}(T)=\sqrt{\frac{\sum_{t \in T}(t-\bar{T})^{2}}{|T|-1}},
$$

where $\bar{T}$ is the average value of temperatures in $T,|T|$ is the number of temperatures in $T$.

1.3. Deviation of temperature values relative to the average

$$
F_{3}(T)=\max _{t \in T}|\bar{T}-t|
$$

1.4. Deviation of internal gradients. The maximum and minimum values, (2), (3), (4), and the following $L^{p}$ norms are used as measures of the spread of internal gradients:

$$
\begin{gathered}
F_{4}(T)=\|T\|_{1}, \\
F_{5}(T)=\|T\|_{2}, \\
F_{6}(T)=\|T\|_{\infty},
\end{gathered}
$$

where

$$
\begin{gathered}
\|T\|_{p}=\left(\sum_{t \in T}|t|^{p}\right)^{\frac{1}{p}}, \\
\|T\|_{\infty}=\max _{t \in T}|t| .
\end{gathered}
$$

More specific:

1.4.1. Maximum difference between the internal temperatures of the lower leg and the average temperature, standing position

$$
f_{1}\left(x^{i}\right)=F_{3}\left(T^{i, m w, s t}\right)=\max _{t \in T^{i, m w, s t}}\left|\overline{T^{i, m w, s t}}-t\right|
$$

1.4.2. The spread of internal temperatures of the lower leg measured in the lying position

$$
f_{2}\left(x^{i}\right)=F_{2}\left(T^{i, m w, l y}\right)=\sqrt{\frac{\sum_{t \in T^{i, m w, l y}}\left(t-\overline{T^{i, m w, l y}}\right)^{2}}{\left|T^{i, m w, l y}\right|-1}}
$$

1.4.3. Oscillation of the surface temperatures of the lower leg measured in the standing position

$$
f_{3}\left(x^{i}\right)=F_{1}\left(T^{i, i r, s t}\right)=\max _{t \in T^{i, i r, s t}} t-\min _{t \in T^{i, i r, s t}} t
$$

2. Hypothesis about the symmetry of temperature fields, according to which healthy lower legs are characterized by slight deviations of temperatures at the corresponding points (subregions), as well as slight differences in the values of the corresponding characteristics. 
The following characteristics are used as symmetry measures:

$$
\begin{gathered}
F\left(T_{c}, T_{p}\right)=\left\|T_{c}-T_{p}\right\|, \\
F\left(T_{c}, T_{p}\right)=\left\|T_{c}\right\|-\left\|T_{p}\right\|,
\end{gathered}
$$

where $\|z\|$ is a functional, $T_{c}-T_{p}$ is an element-wise difference, $T_{c}$ is current, and $T_{p}$ is paired group of temperatures. These characteristics require an additional step of data preprocessing, as well as the presence of a pair for every lower leg in the dataset. For example, during the preprocessing of lower legs data, if the left lower leg is being viewed at the moment, then current temperature group is internal or surface temperatures of the left lower leg, and paired group is internal or surface temperatures of the right lower leg.

For paired temperature groups, the calculated characteristics are basically defined under the previous hypothesis, e.g:

2.1. Maximum absolute value of temperature difference of the corresponding points

$$
F_{7}\left(T_{c}, T_{p}\right)=F_{6}\left(T_{c}-T_{p}\right)
$$

2.2. Difference between the minimum and maximum temperatures of the lower legs

$$
F_{8}\left(T_{c}, T_{p}\right)=\max _{t \in T_{c}} t-\min _{t \in T_{p}} t
$$

2.3. Difference of standard deviations of lower leg temperatures

$$
F_{9}\left(T_{c}, T_{p}\right)=F_{2}\left(T_{c}\right)-F_{2}\left(T_{p}\right)
$$

2.4. Difference of average values, etc.

$$
F_{10}\left(T_{c}, T_{p}\right)=\overline{T_{c}}-\overline{T_{p}}
$$

3. Hypothesis about the stability of temperature fields, according to which healthy lower legs are characterized by slight differences in temperatures measured in different positions.

Features of this group characterize the degree of similarity between temperature fields in different positions and are practically similar to features defined within the symmetry hypothesis. For example:

3.1. Difference of average values of surface temperatures of the lower leg, measured in standing and lying positions

$$
f_{4}\left(x^{i}\right)=F_{10}\left(T^{i, i r, s t}, T^{i, i r, l y}\right)=\overline{T^{i, i r, s t}}-\overline{T^{i, i r, l y}}
$$

3.2. Maximum absolute value of the difference between the internal temperature gradients of the lower leg, measured in standing and 
lying positions

$$
f_{5}\left(x^{i}\right)=F_{7}\left(T^{i, g, s t}, T^{i, g, l y}\right)=\left\|T^{i, g, s t}-T^{i, g, l y}\right\|_{\infty}
$$

4. Hypotheses related to the physiological structure of the lower legs $[7,8]$. The values of lateral-medial and axial gradients for different groups of temperatures are considered, as well as their differences for the corresponding groups of the right and left lower legs:

4.1. lateral-medial gradient

$$
F_{11}(T)=L M G(T)=\overline{T_{e x t}}-\overline{T_{i n t}},
$$

where $T_{\text {ext }}$ is a subgroup of temperatures of the external part of the lower leg (points $1,4,7,10$ ) and $T_{\text {int }}$ is a subgroup of temperatures of the internal part of the lower leg (points 3,6,9, 12).

4.2. axial gradient

$$
F_{12}(T)=A G(T)=\overline{T_{t o p}}-\overline{T_{b o t}},
$$

where $T_{t o p}$ is a subgroup of temperatures of the top part of the lower leg (points $1,2,3$ ) and $T_{b o t}$ is a subgroup of temperatures of the bottom part of the lower leg (points 10, 11, 12).

Going back to the example in Figure 1, the following is observed:

1. Similarity of the internal and surface temperature fields of the right lower leg when measured standing or lying. Features of the form (6) can be applied for detection and description;

2. Similarity of the internal temperature fields of the right lower leg when measured standing and lying. Similar for surface temperatures. The same features of the form (6);

3. Asymmetry of the temperature fields of the right and left lower leg. Features of the form (6) are applied, including all other features, e.g. (7).

4. Differences in the internal and surface temperature fields of the left lower leg when measured standing or lying. Similar to item 2;

5. Bell-shaped contours are observed in the left lower leg. Such data can be detected by using, for example, deviation measures, features of the form (5), and the axial gradient (8), etc.

\subsection{Thermometric features}

For every object in the dataset, the values of functions $f$ are calculated and 128 new features are constructed. Further, by binarizing [13] the 
obtained values, a set of thermometric features is constructed

$$
S=\left(\phi_{1}, \phi_{2}, \ldots, \phi_{s}\right)
$$

where $s$ is the number of features.

A thermometric feature is a triplet $\phi=(f, I, W)$, where $I$ is an interval and $W$ is a weight (informativeness of $f$ on $I$ ), or a quantitative measure that determines how well a feature separates objects of one class from other classes. Thermometric feature is considered fulfilled (observed for the object $\left.x^{i}\right)$ if $f\left(x^{i}\right) \in I$.

Statistical informativeness [13] was applied for calculating weights. In the case of several classes, it is defined as

$$
I(\phi, X)=-\ln \frac{C_{P_{1}}^{p_{1}} \ldots C_{P_{K}}^{p_{K}}}{C_{m}^{p}},
$$

where $C_{n}^{k}$ is a binomial coefficient, $P_{i}$ is the number of class $i$ objects in sample $X, p_{i}$ is the number of class $i$ objects, for which the feature $\phi$ is observed, $p=p_{1}+\cdots+p_{K}$. This measure is fair enough and works well for small unbalanced datasets.

A key feature of thermometric features is interpretability, which makes it possible to form a conclusion about the state of an object based on the values of thermometric features. Vector $\left(\phi_{1}, \phi_{2}, \ldots, \phi_{s}\right)$ dynamically describes the condition of the object in the sample. Element of a vector with index $j$ equals 1 if feature $j$ is observed for the object $x^{i}$, and 0 otherwise.

After all transformations, the matrix (1) takes the form of a binary matrix

$$
X^{\prime}=\left[\begin{array}{cccc}
\phi_{1}\left(x^{1}\right) & \phi_{2}\left(x^{1}\right) & \ldots & \phi_{s}\left(x^{1}\right) \\
\phi_{1}\left(x^{2}\right) & \phi_{2}\left(x^{2}\right) & \ldots & \phi_{s}\left(x^{2}\right) \\
\ldots \ldots \ldots \ldots \ldots \ldots \ldots \ldots \ldots \ldots \ldots & \ldots \ldots \ldots \\
\phi_{1}\left(x^{m}\right) & \phi_{2}\left(x^{m}\right) & \ldots & \phi_{s}\left(x^{m}\right)
\end{array}\right]
$$

and further the classification algorithms are constructed. Moreover, every feature from (11) can be described in a language understandable to a diagnostician.

As binarization result, a large number of thermometric features can be obtained, while many features do not provide new information in combination with each other, so here arises the problem of feature selection. To solve this problem, logistic regression with $L_{1}$-regularization [14] is applied. The process of transforming the feature space is illustrated in Figure 3. 


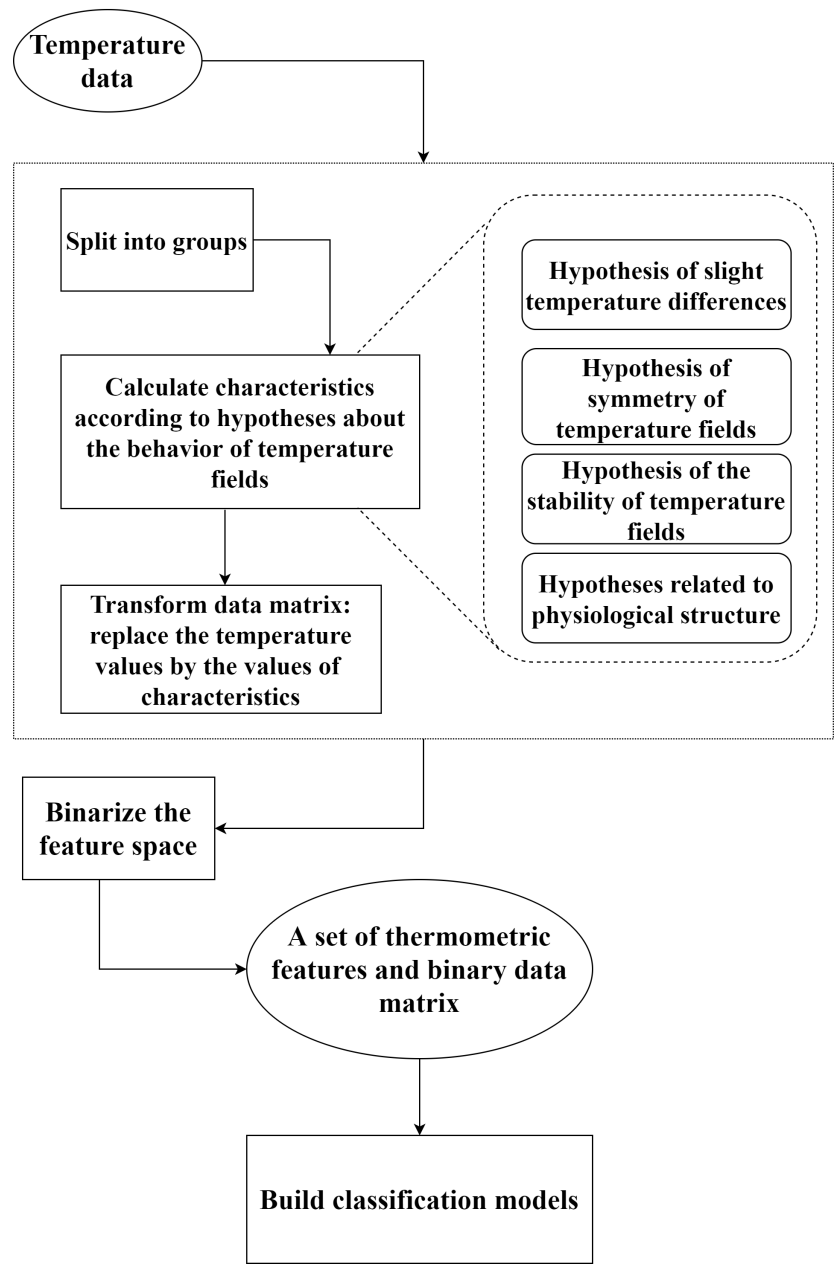

FIgURE 3. Illustration of the process of transforming the feature space.

\subsection{Modeling exercise}

To evaluate the efficiency of thermometric features in solving the problem of differential diagnosis, several classification models are built. The traditional method of logistic regression is applied as the basis for all models. 
Classification algorithm is defined as

$$
a\left(x^{i}\right)=\left\{\begin{array}{l}
1, \text { if } h_{W}\left(x^{i}\right) \geq 0.5, \\
0, \text { otherwise }
\end{array}\right.
$$

where

$$
h_{W}\left(x^{i}\right)=g\left(W_{0}+\sum_{j=1}^{s} W_{j} \phi_{j}\left(x^{i}\right)\right)
$$

is the sum of weights of thermometric features, $W_{j}$ is a weight of feature $\phi_{j}$, and

$$
g(z)=\frac{1}{1+e^{-z}}
$$

is a sigmoid.

Together with thermometric features, logistic regression is a weighted feature voting algorithm. To justify and explain the classification result, it is sufficient to combine the descriptions of the object's features.

The following approaches for solving the multiclass classification problem are considered:

1. Logistic regression (LR), one versus rest (OvR). For every class in the dataset, a model that determines whether an object belongs to the selected class is built. The most confident model determines the result. In total, $C$ classifiers are trained, $C$ is the number of classes.

2. LR, one versus one (OvO). For every pair of classes in the dataset, a separate classification model is built. A majority vote determines the result. In total, $\frac{C(C-1)}{2}$ classifiers are trained.

3. Multinomial logistic regression (MLR), which is a generalization of logistic regression for the case of several classes.

In addition to multiclass classification, a hierarchical approach is also considered. The first model is applied for solving binary classification problem: for separating Healthy class from others, which can be done quite effectively. And the second model is applied to clarify the class of disease.

Stratified nested cross-validation [15] is used to evaluate the efficiency of classification and to compare models with each other. The dataset is split into 9 blocks at the outer level and 8 blocks at the inner level. Balanced precision [16] is used as a performance metric. It is defined as the 
TABLE 1. Classification performance

\begin{tabular}{ccclcllll}
\hline \multicolumn{2}{c}{ Metric } & \multicolumn{2}{c}{ LR, OvR } & \multicolumn{2}{c}{ LR, OvO } & \multicolumn{2}{c}{ MLR } \\
& & & w/o H & & & w/o H & & w/o H \\
\hline \multirow{2}{*}{ Acc $b$} & Avg & 0.557 & 0.548 & 0.574 & 0.537 & 0.56 & 0.541 \\
& Std Dev & 0.102 & 0.074 & 0.065 & 0.05 & 0.073 & 0.078 \\
Recall $_{0}$ & Avg & 0.844 & - & 0.781 & - & 0.838 & - \\
& Std Dev & 0.136 & - & 0.162 & - & 0.096 & - \\
Recall $_{1}$ & Avg & 0.609 & 0.641 & 0.583 & 0.684 & 0.597 & 0.608 \\
& Std Dev & 0.138 & 0.176 & 0.13 & 0.092 & 0.12 & 0.151 \\
Recall $_{2}$ & Avg & 0.614 & 0.579 & 0.617 & 0.549 & 0.595 & 0.641 \\
& Std Dev & 0.197 & 0.142 & 0.09 & 0.11 & 0.163 & 0.167 \\
Recall $_{3}$ & Avg & 0.45 & 0.576 & 0.535 & 0.561 & 0.519 & 0.519 \\
& Std Dev & 0.18 & 0.136 & 0.104 & 0.189 & 0.143 & 0.177 \\
Recall $_{4}$ & Avg & 0.271 & 0.396 & 0.354 & 0.354 & 0.25 & 0.396 \\
& Std Dev & 0.333 & 0.249 & 0.227 & 0.227 & 0.204 & 0.249 \\
\hline
\end{tabular}

average value of recall for every class:

$$
\begin{aligned}
& \operatorname{Acc}_{b}=\sum_{i=1}^{C} \frac{\text { Recall }_{i}}{C}, \\
& \operatorname{Recall}_{i}=\frac{\text { correct }_{i}}{\text { total }_{i}},
\end{aligned}
$$

where correct $_{i}$ is the number of class $i$ objects, which are classified as $i$, total $_{i}$ is the total number of class $i$ objects, $C$ is the number of classes.

\section{Results and discussion}

Results are presented in Table 1. There the mark "w/o H" belongs to algorithms that are trained on the dataset without class Healthy, Avg is the average score, Std Dev is standard deviation. The highest balanced accuracy scores are achieved using $\mathrm{LR}, \mathrm{OvO}$ in the dataset containing all classes, and using LR, OvR in the dataset without class 0 (Healthy).

$\mathrm{LR}, \mathrm{OvR}$ is the best model for identifying class Healthy. It has an average accuracy of 0.844 .

Variance of LR, OvO scores is less than that of other models. This model, on average, performs better than other models in identifying class 2 , which characterizes venous diseases. However, other models have better performance in identifying the rest of classes.

Models built for the dataset without class 0 have higher classification accuracy for various diseases than the same algorithms for the full dataset. In that case, LR, OvR model is leading and more efficiently identifies 
TABLE 2. Thermometric features

\begin{tabular}{lllllll}
\hline Feature & $\mathrm{W}$ & $R_{0}$ & $R_{1}$ & $R_{2}$ & $R_{3}$ & $R_{4}$ \\
\hline$\overline{T_{c}^{i, i r, s t}}-\overline{T_{p}^{i, i r, s t}} \in(-\infty,-0.288)$ & 48.32 & 0.0 & 0.7 & 0.18 & 0.16 & 0.2 \\
$\left\|T_{c}^{i, i r, s t}-T_{p}^{i, i r, s t}\right\|_{2} \in(1.967,7.72)$ & 44.91 & 0.0 & 0.75 & 0.64 & 0.62 & 0.85 \\
$\left\|T_{c}^{i, m w, l y}-T_{p}^{i, m w, l y}\right\|_{1} \in(4.25, \infty)$ & 44.65 & 0.0 & 0.69 & 0.57 & 0.55 & 0.95 \\
$\overline{T_{c}^{i, i r, s t}}-\overline{T_{p}^{i, i r, s t}} \in(-0.288,0.279)$ & 44.44 & 1.0 & 0.25 & 0.48 & 0.39 & 0.15 \\
$\left\|T_{c}^{i, m w, l y}-T_{p}^{i, m w, l y}\right\|_{1} \in(1.45,3.85)$ & 42.81 & 0.94 & 0.22 & 0.34 & 0.32 & 0.05 \\
$\left\|T_{c}^{i, i r, l y}-T_{p}^{i, i r, l y}\right\|_{1} \in(4.45, \infty)$ & 42.61 & 0.17 & 0.85 & 0.64 & 0.9 & 0.85 \\
$\left\|T_{c}^{i, i r, s t}-T_{p}^{i, i r, s t}\right\|_{\infty} \in(1.25, \infty)$ & 41.62 & 0.0 & 0.73 & 0.64 & 0.54 & 0.7 \\
$\overline{T_{c}^{i, i r, l y}}-\overline{T_{p}^{i, i r, l y}} \in(0.221, \infty)$ & 40.39 & 0.11 & 0.04 & 0.38 & 0.54 & 0.7 \\
$\overline{T_{c}^{i, m w, l y}}-\overline{T_{p}^{i, m w, l y}} \in(-\infty,-0.096)$ & 39.98 & 0.14 & 0.75 & 0.31 & 0.16 & 0.25 \\
$\left\|T_{c}^{i, m w, s t}-T_{p}^{i, m w, s t}\right\|_{1} \in[0,5.55)$ & 39.93 & 1.0 & 0.49 & 0.67 & 0.62 & 0.05 \\
\hline
\end{tabular}

class 3. In comparison with MLR, this model distinguishes class 1 better, class 2 worse, and class 3 with the same accuracy. In comparison with $\mathrm{LR}, \mathrm{OvO}$, this model identifies class 1 worse and better identifies all other classes. Class 4 is characterized by a significant variance of average accuracy estimates.

Table 2 shows examples of the most informative thermometric features, which are the basis for classification models. There $W$ is informativeness, $R_{i}$ is a proportion of class $i$ objects that have a feature. All these features describe the symmetry of temperature fields of the lower legs.

Three features with the highest informativeness are not observed in Healthy class. They allow effective detection of the lower legs with diseases. Such features are the difference in means and the deviation of surface temperatures of the legs measured in standing position, as well as the deviation of internal temperatures measured in the lying position.

Almost all healthy lower legs are characterized by a small difference between average values of skin temperatures measured in the standing position and a small deviation of internal temperatures measured in both standing and lying positions.

In class 1 , there is practically no high difference in average values of internal or surface temperatures measured both standing and lying. At the 
same time, the difference between average values of surface temperatures in standing position is usually higher for them than for class 0 .

Class 4 does not exhibit low deviance of internal temperature gradients measured in the lying position. A low deviation of internal temperatures, measured both standing and lying, is practically not observed.

These and other features are used in weighted voting classifiers. And the given features signal that different classes of diseases are characterized by high deviation of internal and surface temperatures of the lower legs.

\section{Conclusion}

The most effective universal algorithm for solving the task is LR, OvO. It has an average balanced accuracy of 0.574 . However, when applying a hierarchy of classifiers and reducing the problem to a binary classification Healthy/Sick with subsequent clarification of the disease, the best result can be achieved with LR, OvR. It has an average estimate of clarification of the disease class of 0.548 .

Earlier, Vedenyapin and Losev [12] applied three two-layer neural networks in sequential order to solve the differential diagnosis problem. Every network separated one of the classes from all the others, and the rest were classified as Healthy. That approach has an accuracy of 0.59. A detailed comparison of results is not possible because evaluation methods and datasets are a bit different. Nevertheless, the presented approach has the following advantages over neural networks:

1. A possibility to justify and explain the classification result. Every thermometric feature can be interpreted;

2. Anamnesis data (indicators of edema, pain, skin changes) is not used. It is possible that adding anamnesis data to features space can significantly improve the performance of classification. However, this is of interest for further research.

Results show the applicability of the model for dynamically describing the patient's condition in differential diagnosis of venous diseases. The key feature of constructed algorithms is the possibility to justify and explain the diagnostic decision.

\section{References}

[1] Roadmap for the development of "Pass-through" digital technology "Neurotechnologies and Artificial Intelligence", Ministry of Digital Development, 
Communications and Mass Media of the Russian Federation, 2019 (in Russian). (URL) $\uparrow_{37}$

[2] M. Sedankin, A. Gudkov, V. Leushin, S. Vesnin, I. Sidorov, D. Chupina, S. Agasieva, V. Skuratov, S. Chizhikov. "Microwave radiometry of the pelvic organs", Biomedical Engineering, 53:4 (2019), pp. 288-292. do $\uparrow_{38}$

[3] A.V. Tarakanov, A.A. Tarakanov, S. Vesnin, V. Efremov, N. Roberts, I. Goryanin. "Influence of ambient temperature on recording of skin and deep tissue temperature in region of lumbar spine", European Journal of Molecular \& Clinical Medicine, 7:1 (2020), pp. 21-26. do $\uparrow_{38}$

[4] I. Goryanin, S. Karbainov, O. Shevelev, A. Tarakanov, K. Redpath, S. Vesnin, Y. Ivanov. "Passive microwave radiometry in biomedical studies", Drug Discovery Today, 25:4 (2020), pp. 757-763. do $\uparrow_{38}$

[5] T.V. Zamechnik, S.I. Larin, A.G. Losev. Combined radiothermometry as a method for studying venous circulation of the lower extremities, monograph, VolgGMU Publishing House, Volgograd, 2015, ISBN 978-5-9652-0400-7 (in Russian), 252 pp. $\uparrow_{38}$

[6] T.V. Zamechnik, N.S. Ovcharenko, S.I. Larin, A.G. Losev. "Reliability assessment of combined thermography as a method for the characteristic of the lower limb venous system", Flebologiya. Journal of Venous Disorders, 4:3 (2010), pp. 23-26 (in Russian). * URL $\uparrow_{38}$

[7] E.V. Anisimova, T.V. Zamechnik, A.G. Losev. "About one mathematical model of diagnostics of venous diseases according to the combined thermometry", Bulletin of new medical technologies, 19:2 (2012), pp. 239-241 (in Russian). (URL) $* \uparrow_{38,44}$

[8] T.A. Stavrov, E.V. Bukina, A.G. Losev, T.V. Zamechnik. "Mathematical verification of early recurrence of the varicose disease after the endovascular laser obliteration of the great saphenous vein according to data of the radiothermometry", Bulletin of new medical technologies, 20:2 (2013), pp. 14-18 (in Russian). (URL) $\mathcal{*}_{3} \uparrow_{38,44}$

[9] A.G. Losev, V.V. Levshinsky. "The thermometry data mining in the diagnostics of mammary glands", Large-Scale Systems Control, 70 (2017), pp. 113-135 (in Russian). 回 $*_{38,41}$

[10] V. Levshinskii. "Intelligent system for diagnostics of venous diseases based on the microwave radiothermometry data", "Smart Technologies" for Society, State and Economy, ISC 2020, Lecture Notes in Networks and Systems, vol. 155, eds. E. G. Popkova, B. S. Sergi, Springer, Cham, 2021, pp. 212-219. dof $\uparrow_{38,41}$

[11] E.A. Mazepa, O.V. Grishina, V.V. Levshinsky, H.M. Suleymanova. "The unification of microvawe radio thermometry method", Mathematical Physics and Computer Simulation, 20:6 (2017), pp. 38-50 (in Russian). do $\uparrow_{38}$

[12] D.A. Vedenyapin, A.G. Losev. "Neural networks in vascular diseases diagnosis", Large-Scale Systems Control: collection of works, 39 (2012), pp. 219-229 (in Russian). 回 $\uparrow_{39,50}$ 
[13] K.V. Vorontsov. Lectures on logical classification algorithms, 2007 (in Russian), 53 pp. URL $\uparrow_{44,45}$

[14] P. Flach. Machine Learning. The Art and Science of Algorithms that Make Sense of Data, 1st Edition, Cambridge University Press, 2012, ISBN 978-1107422223, 409 pp. $\uparrow_{45}$

[15] S. Raschka, V. Mirjalili. Python Machine Learning: Machine Learning and Deep Learning with Python, scikit-learn, and TensorFlow 2, 3rd Ed., Packt Publishing, Birmingham, UK, 2019, ISBN 978-1789955750, 770 pp. $\uparrow_{47}$

[16] M. Grandini, E. Bagli, G. Visani. Metrics for multi-class classification: an overview, 2020, 17 pp. arXiv 2008.05756 [stat.ML] $\uparrow_{47}$

$\begin{array}{ll}\text { Received } & 16.03 .2021 \\ \text { Revised } & 24.03 .2021 \\ \text { Published } & 14.04 .2021\end{array}$

Recommended by prof. V.M. Hachumov

Sample citation of this publication:

Vladislav V. Levshinskii. "Multiclass Classification in the Problem of Differential Diagnosis of Venous Diseases Based on Microwave Radiometry Data". Program Systems: Theory and Applications, 2021, 12:2(49), pp. 37-52.

do) $10.25209 / 2079-3316-2021-12-2-37-52$

(URL) http://psta.psiras.ru/read/psta2021_2_37-52.pdf

About the author:

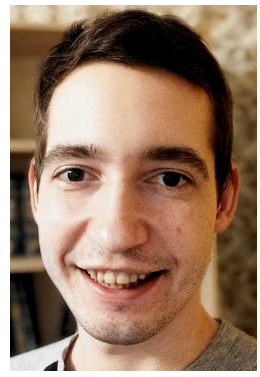

\section{Vladislav Viktorovich Levshinskii}

Postgraduate student of Volgograd State University. Interested in programming, artifical intelligence, data science, machine learning and mathematical modeling.

$$
\begin{array}{ll}
\text { (D) } & \text { 0000-0002-3587-0124 } \\
\text { e-mail: } & \text { v.levshinskii@volsu.ru }
\end{array}
$$

